\title{
Téoros
}

Revue de recherche en tourisme

\section{Les croisières sur le Saint-Laurent... un peu d'histoire}

\section{Luc Tittley}

Volume 14, numéro 2, été 1995

Le tourisme : toute une histoire!

URI : https://id.erudit.org/iderudit/1075094ar

DOI : https://doi.org/10.7202/1075094ar

Aller au sommaire du numéro

Éditeur(s)

Université du Québec à Montréal

ISSN

0712-8657 (imprimé)

1923-2705 (numérique)

Découvrir la revue

Citer cet article

Tittley, L. (1995). Les croisières sur le Saint-Laurent... un peu d'histoire. Téoros, 14(2), 12-14. https://doi.org/10.7202/1075094ar d'utilisation que vous pouvez consulter en ligne.

https://apropos.erudit.org/fr/usagers/politique-dutilisation/ 


\section{Les croisières sur le Saint-Laurent... un peu d'histoire}

Luc Tittley*

- L'avènement de la machine à vapeur au tournant du $\mathrm{XIX}^{e}$ siècle marque une véritable révolution dans le transport maritime comme dans le transport terrestre: on se libère du vent, ce qui était d'autant plus utile sur un fleuve Saint-Laurent marqué par des basfonds, les courants et la marée.

- Alors qu'on se déplaçait jusqu’alors par nécessité, il ne fallut qu'une trentaine d'années à la bourgeoisie marchande etindustrielleémergente pour découvrir le plaisir du voyage d'agrémentetles paysages fabuleux du SaintLaurent. C'est ainsi que naquit, vers 1830, la croisière sur le Saint-Laurent en navire à vapeur. Ce produit touristique devait s'avérer populaire pendant plus d'un siècle. En effet, cen'est qu'en 1965 que les trois derniers 4bateaux blancs» de la Canada Steamship Line furent retirés du fleuve.

- Nées à la faveur de l'effervescence économique du XIX ${ }^{e}$ siècle qui suscita la création de grands empires industriels et commerciaux à Montréal et en Nouvelle-Angleterre, les croisières fluviales ont prospéré dans la fouléedu romantisme del'èrevictorienne, alors que se développaient les destinations de villégiature de Charlevoix et du Bas-du-Fleuve. Elles ont disparu avec la généralisation de l'automobile et l'avion.

\section{Le transport fluvial \\ sur le Saint-Laurent}

- Dès sa mise en service, à la fin du XVIII" siècle, le bateau à vapeur allait connaittre une popularité phénoménale partout dans le monde. Cette nouvelle technologieoffrait un navire plus *manoeuvrants etpl us autonome que tous les navires à voile utilisés jusqu'alors. Elle devait déclencher une activité souvent fébrilesur le Saint-

Monsieur Lue Tittley, geographe of urbaniste, est. consultant en tourisme et vice-president du bureau d'études Daniel Arbour \& Associes.

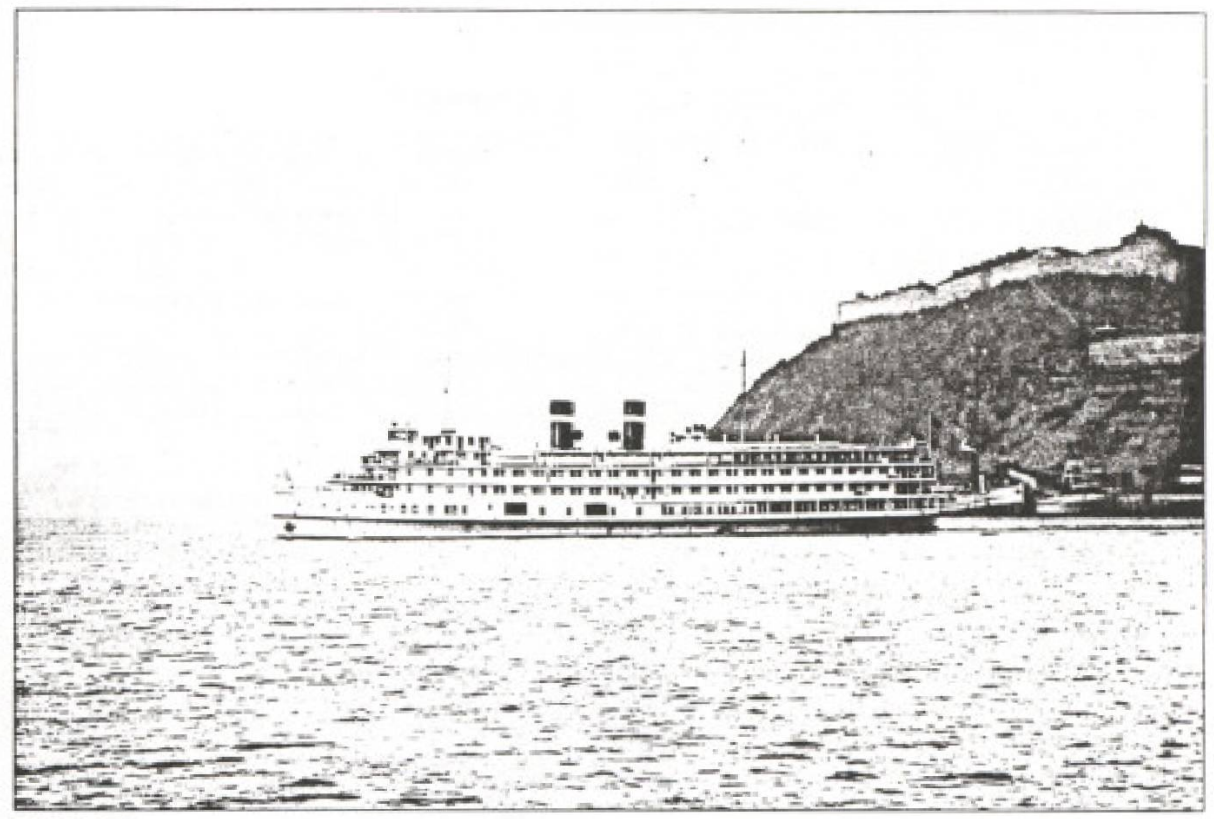

Le SS St-Lawrence en face de Québec vers 1940.

Photo: Canada Stcamship Lines.

Laurent, en particulier entre Montréal et Québec. Les nouveaux navires à vapeur permettaient de vaincre les difficultés de la navigation sur le Saint-Laurent en amont de Québec et d'assurer un service régulier, fiable et rapide.

- C'est en 1809 que John Molson met en service le premier vapeur, le Accomodation, entre Montréal et Québec. Le succès vient très rapidement et une flotte se constitue sous le nom de la St. Lawrence Steam Navigation Company.

- Les compétiteurs se multiplient. Molson trouve un compétiteur particulièrement entreprenant dans la Torrance Line de David Torrance... mais également dans la Compagnie du Richelieu fondée par Joseph-Félix Sincennes. Ce dernier, après des débuts modestes sur le Richelieu s'attaquait maintenant à la route du SaintLaurentavec des associésmontréalais, des marchands francophones (notamment Victor Hudon de Hudon Coton Mills), et deuxnavires de grande qualité, le Napaléon et le Victoria, construits au chantier naval Cantin à Montréal.

- La compétition entre les nombreuses compagniesde navigation sur le SaintLaurent allait entraîner une guerre des prix qui mena les plus faibles à la faillite On vit également des courses devapeurs mémorables qui n'ont pris fin qu'avec la tragédie du Montrealer de la Torrance Line. 253 personnes, surtout des immigrants en route vers Montréal, ont perdu la vie, le 26 juin 1857 , lorsque le navire en flamme fut échoué trop loin du bord suite à une course meurtrière avec le Napolén de la Compagnie du Richelieu. Cette tragédie conduisit à l'a bsorption de la Torrance par la Compagnie du Richelieu.

- Des ententes de partage de marchés (avec la Royal Mail Line qui desservait le Haut-Canada ) et d'autres acquisitions ( $\mathrm{La}$ Compagnie de la Rive Nord du Saint-Laurent, La Société de Navigation de Terrebonne et l'Assomption, La Compagnie de Navigation du Lac Saint-Pierre) devaient consolider la position de la Compagnie du 
Richelieu. On ajoute à la flotte de nouveaux navires très modernes, le Montréal (1861), le Québec (1865) et le Canada (1867) durant les années 1860.

- Le mouvement de consolidation devait se poursuivre avec des ententes de partage de marchés avec la Canadian Steam Navigation Company de Sir Hugh Allan desservait le SaintLaurent en amont de Montréal et le lac Ontario. Cette entreprise devait éventuellement s'intégrer à la Compagnie de Richelieu en 1874 et donner naissance à la Richelieu and Ontario Navigation Company. Sous lagouvernedeLouis-AdélardSénécal, la $\mathrm{R} \& \mathrm{O}$ acquit la Saguenay Line en 1886 et put ainsi assurer un service intégré de Toronto au Saguenay sur une distance de quelque 800 milles.

- L'emprise de la R \& O continua de s'affirmer sous la présidence du plus puissant homme d'affaires canadienfrançais de l'époque, Louis-Joseph Forget. Celui-ci accorda une place plus importante aux services aux passagers sur l'ensemble du réseau er notamment aux croisières sur le SaintLaurent. En 1898, il fait agrandir de vieil Hôtel Tadoussac et, l'année suivante, il fait construire le premier Manoir Richelieu. Cette infrastructure hôtelière lui permit de concurrencer plus efficacement son grand rival, la St-Lawrence Steam Navigàtion Company de la famille.Molson et de prendre le leadership du marché des croisières.

- Le rêve de créer un transporteur fluvial intégré desservant l'ensemble $\mathrm{du}$ réseau des Grands Lacs à l'Atlantique fut réalisé par Grant Morden. Les Grands Lacs étaient desservis par des entreprises qu'il contrôlait directement ou indirectement comme la Inland Lines, la Great Lakes Navigation Company et la Northern $\mathrm{Na}$ vigation Company. Avec l'appui financier de la compagnie britannique Furness Steamship Company, Grant Morden réussit à amalgamer les trois entreprises des Grands Lacs et, par la suite, à prendre le contrôle de la $\mathrm{R} \&$ \& O. Cette prise de contrôle abouti à la création, en 1913, de la Canadian Steamship Lines, le super-transporteur canadien dont les navires aux cheminées cerclées de noir, de blanc et de rouge naviguent toujours sur le Saint-Laurent et sur toutes les mers du monde.

\section{Voyager sur les navires à vapeur pour le plaisir}

- Les navires à vapeur qui sillonnaient le Saint-Laurent ont toujours transporté des passagers. Outre les habitants des localités situées le long du fleuve, on y rencontrait desindustriels oude riches marchands, des banquiers et autres hommes d'affaires de Montréal et de Québec, mais également des familles riches de Philadelphie, de Boston et de New York. Les navires à vapeur permettaient d'accéder, entre autres, aux lieux de villégiature des bien nantis, qui avaient nom Murray Bay (La Malbaie), la *Newport du Nord», Kamouraska, Rivière-duLoup, Cacouna, Trois-Pistoles, SaintFabien, Sainte-Luce. On pouvait, à l'occasion, y rencontrer des poètes comme Arthur Buies ou des atour operatorsm de l'époque comme Gustave Leve, accompagné d'un de ses Leve's Grand American Excursion Party de New York. Tous étaient attirés par les paysages grandioses et romantiques, les bains de mer revigorants et l'aventure de la découverte des coutumes du pays.

- C'est vers 1830 que les excursions de plaisance sont offertes sur le Watcrloo de la St-Lawrence Steam Navigation Company. La construction d'infrastructures d'accueil adéquates, comme le quai sur pilotis de Pointe-au-Pic (1853), permettent bientôt la mise en place d'un service régulier entre Québec er le ford du Saguenay.

- On met en marché des croisiêres de trois jours depuis Québec. Le navire Sagutenay du capitaine René Simard longe la côtesud del'île d'Orléans, fait escale à Rivière Ouelle, traverse à La Malbaie, revient à Rivière-du-Loup pour la nuit avant de s'engager le lendemain dans le plus beau fjord d'Amérique jusqu'à Chicoutimi.

- En 1865, la Compagnie du Richelieu fait construire, à Sorel, le Quebec, un magnifique navire à roues à aubes, puis en 1870 le Montráal,qui conduisent les passagers de Montréal à Québec d'où ils s'embarquent sur d'autres vapeurs comme le Union, le Saguenay, le St-Larurence ou le Chyde qui, en six heures, relient La Malbaie. Ces navires à deux ponts offrent des salons et des cabines spacieuses, luxueusement aménagées. On y mange très bien et on ne se lasse pas d'admirer les beautés du paysage.

- A partir de 1875, des croisières sont offertes de Toronto jusqu'au Saguenay grâce à la fusion qui a donné naissance à la Richelieu and Ontario Navigation Company qui devait rapidement acheter la Saguenay Line. Dix-huit navires battent son pavillon. On peut loger dans les hôtels de la compagnie: le luxueuxManoir Richelieu etl'Hôtel Tadoussác, davantage axé sur la famille.

- La fusion plus large qui crée la Canada Steamship Lines en 1913 permettra d'offrir, sur les navires de la même compagnie, des croisières depuis le lac Supérieur jusqu'à l'Atlantique. Le produit touristique Saint-Laurent se diversifieets'élargit, alors que la CSL, sous la gouverne de son président William Coverdale, un supporter enthousiastede la vocation touristique de la compagnie, réalise des alliances stratégiques avec les compagnies ferroviaires et s'embarque résolument dans l'amélioration de son propre réseau hôtelier.

\section{Les palais flottants} du début du $X X^{*}$ siècle

- Philippe Dubé les décrit ainsi dans son livre, Deux cents ans d'histoire de la villégiature dans Charlevoix:

Le décor de ces navires suit le goutt élégant de la mode victorienne. $D e$ grands escaliers descendent sur un immense salon feutré d'un tapis couleur bourgogne et couvert d'un dôme richement orné de dorures. Le Montréal par exemple (leplus grand vapeur du Canada: 750 passagers), a son salon paré de bas-reliefs de bronze qui représentent les quatre saisons. Néanmoins, la simplicité est de mise dans les cabines des vapeurs qui peuvent loger près de trois cents passagers. Les extérieurs sont en blanc, ce quileur vaut d'être nonmés abateaux" blanoss par les riverains, qui les voient passer comme des cygnes glissant aver grâce sur londe fluviale.

- Cette description s'applique au plus grand navire passager du Canada. Construit à Toronto en 1903 , le Montréal ransportait 750 passagers avant d'être détruit par les flammes en 
1926 près de Sorel. La tradition des palais flottants fut toutefois maintenue sur les abateaux blancs exploités par la CSL jusqu'en 1965. Avec l'ćvolution de la technologie, les vapeurs a roues à aubes furent progressivement remplacés par des vapeurs à hélices, plusmodernes et plus petits, construits expressément pour les croisières sur le Saint-Laurent et le Saguenay, tels le Richelieu, le TadoucracetleSt-Lawrence.

- L'approche de la CSL, pour la publicité et la promotion de ses croisières, avait quelquechose delyriqueetmême de poétque. Plus qu'un mode de transport, on offrait une expéricnce d'évasion dans l'histoire et dans le pittoresque. L'ontreprise engageait des auteurs chargés d'écrire des guides touristiques décrivant le parcours. Elle publiait également des livtes commandés à des auteurs réputés comme Blodwen Davies (Saguenay, The River of Deep Water), joliment reliés et illustrés, qu'on vendait dans les kjosques à bord des navires et dans les hôtels de la compagnie. Le président de la compagnie lui-même, William Cloverdale, füt l'auteur d'un de ces livres: Tadoussac Then and Now: A History and Narrative of The Kingdom of The Saguenay.

- La CSL accordait une attention particulière à la qualité de son produit. Ses abateaux blancss étaient spectaculaires, d'une propreté impeccable. Le personnel était stylé et habillé... de blanc. On offrait plus que desvoyages à horaires fixes dans des paysages exceptionnels. Les navires étaient de véritables centres de loisirs avec une restauration de qualité, des programme d'animation, dela danse avec orchestre, des jeux de société, des concours et des excursions à terre. Les hốtels de la compagnie étaient équipés de terrains de golf, de piscines d'eau de mer chauffée, de tennis, de salles de danse.

- Le président Cloverdale de la CSL avait choisi de meubler le Manoir Richelieu d'une collection exceptionnelle d'antiquités, d'artefacts et de peintures, de cartes anciennes, d'estampes originales illustrant l'histoire du Canada, de peintures contemporaines représentant des scènes canadiennes d'autrefois. À Tadoussac, la CSL reconstruisit mème le poste de traite de Pierre Chauvin sur les fondations datant de 1600 . pour en faire un musée La production des artisans de Charlevoix était vendue sur les bateaux et dans les hootcls. Elle était utilisée dans la décoration et donnait lieu à une exposition annuelle au Manoir Richelieu.

\section{Sauter les rapides en navire à vapeur}

- Pour le touriste désireux de faire le trajet complet depuis Toronto, un changement denavires'imposait pour le passage des rapides du Long Sault ęt de Lachine, en amont de Montréal. A Prescott, les passagers montaient à bord du Rapids King, du Rapids Queen ou du Rapils Prince, des vapeurs à faibletirant d'eau ( 5 pieds). Nul doute que wsauter les rapides* constituait le point fort de la croisière TorontoMontréal. Comme le relate Edward Andrew Collard dans son livte sur l'histoire de la CSL:

Of all The sigbts and scenes on The long passenger route, notbing equaled The brief spasm of excitement in sbooting The Lacbine Rapids. It offered The intense stimulation that comes from deliberately skirting danger. The descent was safe only because of The pilot's skill.

The sbooting of The Long Sault Rapids farther upstream was a prelude to The deeper adventure in The Lacbine. The Long Sault bad The roar of white Water, but was a gradual descent, spread over nine miles. It lacked The wilder turbulence, The steeper drops, The more abrupt twist of Tbe Lacbine.

As Tbesbipappraacbed The Lachine Rapids passengers began to bave second thoughts. But they were conmitted; they could not ask The captain to let them off.

\section{Le déclin}

- Le romantisme de l'ère victorienne s'éteignit avec la Première Guerre mondiale. Puis la Dépression contribua à réduire conșidérablement le nombre de passagers sur les abatea ux blancs dela CSL. La Seconde Guerre mondiale stimula certes l'économie, mais monopolisait totalement l'industrie du transport maritime.

- En 1944, William Cloverdale, le président de CSL, annonce à son conseil d'administration, un peu médusé, qu'il faisait du trafic passager sa priorité absolue. Les immobilisations de la compagnie seraient concentrées dans ce secteur, au détriment même dẹs autres intérêts de l'entreprise. Il s'agissait là d'une mauvaise décision d'affaires selon Edward Andrew Collard:

The passenger trade, though glamourous, was doomed. The world after Tbe war would be radically different from what It bad been before. Development in technology, stimulated by The needs of war, would Now be applied to civilian life and would change its babits. The growth of air travel, plus The proliferation and convenience of The automobile, aided by The improvement of bighways, would make steamboat travel on Canada's Inland waterwaysseent tame, old-fasbioned and geograpbically limited.

- La mort de Cloverdale, quelques années plus tard, conduisit à une révision de ces orientations. La CSL maintint tout de même ses «bateaux blancs* en service jusqu'en 1965. Mais le nombre de passagers n'avait cessé de diminuer, les navires avaient vieilli et étaient devenus dangereux. La mise aux normes ou le remplacement des navires exigeait des investissements énormes, impossibles à rentabiliser. L'annonce de la mise en rancart des navires etla findu service par le conseil d'administration de la CSL tomba sur le fil de presse le 10 novembre. Au cours des trois ans qui suivirent, la CSL vendait le Manoir Richelieu et l'Hôtel Tadoussac.

\section{RUEFÉRENCES}

AGASSIZ, Garnault, Niagara to The Sea, Canada Steamship Lines limited, Montreal, 1915, 132 p. COLLARD, Edward Andrew. Passage to The Sea. The Story of Canada Steamship Lines, Doubleday Canada limited, Toronto, 1991, 432 p.

DAVIES, Blodwen, Smguenay, The River of Deep Waters, Canada Steamship Lines limited, Montreal. 1930, $204 \mathrm{p}$.

DUBE, Philippe, Deux cents ans de villegiature dans Char levobx, Quebec, Les Presses de rUniversite Laval $1986,336 \mathrm{p}$ 